\title{
Sensibilidade ao contraste na retinopatia diabética tratada por panfotocoagulação com laser de argônnio
}

\author{
Contrast sensitivity in diabetic retinopathy treated with \\ argon laser panphotocoagulation
}

\author{
Otacílio de Oliveira Maia Júnior ${ }^{1}$ \\ Walter Yukihiko Takahashi ${ }^{2}$ \\ Marcos Wilson Sampaio ${ }^{3}$ \\ Kenzo Hokazono ${ }^{4}$ \\ Alexandre Kazuo Misawa ${ }^{5}$
}

\begin{tabular}{|l|}
\hline RESUMO \\
\hline Objetivo: Avaliar a sensibilidade ao contraste na retinopatia diabética \\
(RD) tratada por panfotocoagulação com laser de argônio. Métodos: \\
Estudo prospectivo de portadores de retinopatia diabética e acuidade \\
visual de $20 / 20$, tratados com panfotocoagulação retiniana, conforme \\
critérios do ETDRS. Os pacientes foram submetidos, inicialmente, a \\
exame oftalmológico completo e teste de sensibilidade ao contraste \\
(Vision Contrast Test System). Após 3 meses do tratamento, foram \\
reavaliados por meio da acuidade visual e sensibilidade ao contraste. \\
Resultados: A amostra foi composta por 28 pacientes (28 olhos), todos \\
portadores de diabetes tipo 2 . A idade variou entre 45 a 77 anos (média \\
de $57,8 \pm 8,0$ ), sendo 19 pacientes (67,9\%) do sexo masculino e 9 (32,1\%) \\
do feminino. Quanto ao tipo de retinopatia, 18 (64,3\%) apresentavam RD \\
proliferativa e 10 (35,7\%), RD não proliferativa muito grave. Não foi \\
observada nenhuma alteração na acuidade visual pós-tratamento. Quanto \\
à sensibilidade ao contraste, não houve alteração entre o pré e pós- \\
tratamento em todas freqüências espaciais avaliadas: 1,5 (p=0,191); 3,0 \\
(p=0,850); 6,0 ( $p=0,374) ; 12,0$ (p=0,674) e 18,0 (p=0,443). Conclusão: \\
Não foi evidenciada alteração significante na sensibilidade ao contraste \\
de portadores de retinopatia diabética após panfotocoagulação com laser \\
de argônio no período estudado.
\end{tabular}

Descritores: Retinopatia diabética; Retinopatia diabética/terapia; Coagulação por laser; Diabetes mellitus/complicações; Acuidade visual

gia do Hospital das Clínicas da Faculdade de Medicina da Universidade de São Paulo - USP - Setor de Retina e Vítreo - São Paulo (SP) - Brasil.

Pós-Graduando da Faculdade de Medicina da Universidade de São Paulo - USP - São Paulo (SP) - Brasil.

${ }^{2}$ Doutor, Médico Assistente e Chefe do Setor de Retina e Vítreo do Departamento de Oftalmologia do Hospital das Clínicas da Faculdade de Medicina da USP - São Paulo (SP) - Brasil.

${ }^{3}$ Doutor, Médico Assistente e Chefe do Setor de Visão Subnormal do Departamento de Oftalmologia do Hospital das Clínicas da Faculdade de Medicina da USP - São Paulo (SP) - Brasil.

${ }^{4}$ Médico Residente do Departamento de Oftalmologia do Hospital das Clínicas da Faculdade de Medicina da USP - São Paulo (SP) - Brasil.

Estagiário do Departamento de Oftalmologia do Hospital das Clínicas da Faculdade de Medicina da USP - São Paulo (SP) - Brasil.

Endereço para correspondência: Otacílio de Oliveira Maia Júnior. Rua Adelmário Pinheiro, 10 - Salvador (BA) CEP 41900-540 - E-mail: omaiausp@uol.com.br

Recebido para publicação em 14.01.2007

Aprovação em 11.06.2007

\section{INTRODUÇÃO}

A retinopatia diabética $(\mathrm{RD})$ é uma importante causa de perda na acuidade visual e, de acordo com o "Early Treatment Diabetic Retinopathy Study" (ETDRS), a panfotocoagulação retiniana com laser de argônio deve ser considerada em portadores de RD não proliferativa grave e na RD proliferativa, podendo evitar perda visual em casos selecionados e estabilizar a progressão da doença ${ }^{(1-2)}$. Estudos prévios demonstraram que 25 a $43 \%$ dos olhos com RD proliferativa tratados com panfotocoagulação desenvolvem edema macular e distúrbios visuais ${ }^{(3-4)}$. Complicações como alterações no campo visual ${ }^{(4)}$ e na adaptação ao escuro ${ }^{(5-6)}$, diminuição da acuidade visual $^{(7)}$ e decréscimo nas amplitudes do eletroretinograma ${ }^{(8)}$ foram relatadas.

A quantificação mais comum de visão espacial é a determinação da acuidade visual por meio da tabela de Snellen, na qual o indivíduo visualiza letras que tem duas características básicas: tamanho e contraste ${ }^{(9)}$. O
\end{abstract}


teste avalia qual o menor tamanho de letra que é identificado, mantendo-se o contraste alto e constante das letras pretas em relação ao fundo branco em que se situam. Contudo, o grau de visibilidade de uma figura genérica pode ser alterado, reduzindo seu contraste até um limite abaixo do qual não é mais reconhecida, independente do seu tamanho ${ }^{(10)}$. Os testes de sensibilidade ao contraste baseiam-se no conceito de função de sensibilidade ao contraste, que é a capacidade de perceber pequenas variações de luminosidade entre regiões que não apresentam bordas nítidas de separação. Esta importante propriedade de discernir contraste é distinta do poder de discriminação aferido pelas medidas convencionais da acuidade visual. Portanto, a habilidade do indivíduo perceber detalhes de objetos é determinada em grande parte como o mesmo distingue contrastes ${ }^{(11)}$. Por outro lado, pacientes com limitações na capacidade de diferenciar contrastes podem ter dificuldades em diversas situações do cotidiano ${ }^{(10,12)}$.

A panfotocoagulação retiniana pode induzir alteração na acuidade visual e edema macular, acarretando baixa transitória ou permanente da visão ${ }^{(3,12-13)}$. Entretanto, recentemente, não foi observada perda visual significante após panfotocoagulação nos portadores de RD com acuidade de 20/20 e sem edema macular ${ }^{(14)}$.

Como a avaliação da acuidade visual, por meio da tabela de Snellen, fornece uma informação limitada sobre a qualidade de visão e, como mencionado anteriormente, a sensibilidade ao contraste é fundamental para mensurar esta qualidade, o presente estudo objetiva avaliar a sensibilidade ao contraste em portadores de retinopatia diabética com acuidade visual de 20/20, tratada por panfotocoagulação com laser de argônio. Este trabalho faz parte de pesquisa mais ampla a respeito do tema.

\section{MÉTODOS}

Estudo prospectivo de pacientes do Departamento de Oftalmologia do Hospital das Clínicas da Faculdade de Medicina da Universidade de São Paulo (HC-FMUSP), portadores de RD com indicação de panfotocoagulação retiniana, conforme critérios do ETDRS. Outros critérios de inclusão foram: indivíduos de ambos os sexos, qualquer idade, acuidade visual com correção de 20/20, ausência de edema macular, sem tratamento retiniano prévio e sem outras doenças retinianas. Os critérios de exclusão foram: catarata, opacidades na córnea, complicações da RD (hemorragia vítrea e/ou descolamento de retina) ou diminuição da acuidade visual no "follow-up".

Os pacientes foram submetidos, inicialmente, à medida da acuidade visual com melhor correção (tabela de Snellen), teste de sensibilidade ao contraste e a exame oftalmológico completo, incluindo biomicroscopia da mácula. Todos os pacientes foram submetidos à panfotocoagulação retiniana com laser de argônio (Ophthalas 532 Eyelite, Alcon Surgical), segundo padronização recomendada pelo $\operatorname{ETDRS}^{(15)}$ e reavaliados, por meio da acuidade visual e da sensibilidade ao contraste, no terceiro mês do tratamento. Foi considerado apenas um olho por paciente.

O teste de sensibilidade ao contraste utilizado foi o "Vision Contrast Test System" (VCTS 6500, Vistech Consultants Inc). Nesse teste, as freqüências espaciais, representadas em ciclos por graus, medem a sensibilidade do paciente a um objeto de tamanho específico, sendo testadas cinco freqüências: $A=1,5 ; B=3,0 ; C=6,0 ; D=12,0 ; E=18,0$. As baixas freqüências testam objetos de tamanho grande, enquanto as altas freqüências, sensibilidade a pequenos objetos. Cada freqüência testada inicia-se com alto nível de contraste, o qual diminui progressivamente. $\mathrm{O}$ paciente simplesmente relata o "patch" de menor contraste que ele pode enxergar (Figura 1).

A distância da tabela e a iluminação do ambiente foram padronizados pelo Setor de Visão Subnormal, conforme orientação do fabricante. A fim de comparar resultados, os valores padrões de normalidade em candelas $/ \mathrm{m}^{2}$ para cada freqüência espacial são apresentados (Tabela 1).

Inicialmente todas as variáveis foram analisadas descritivamente. Para as variáveis quantitativas, esta análise foi feita por meio da observação dos valores mínimos e máximos e do cálculo de médias e desvios-padrão. Para as variáveis qualitativas, calcularam-se freqüências absolutas e relativas. Para a análise da hipótese de igualdade entre os dois momentos avaliados, utilizou-se o teste não-paramétrico de Wilcoxon para cada parâmetro de sensibilidade ao contraste.

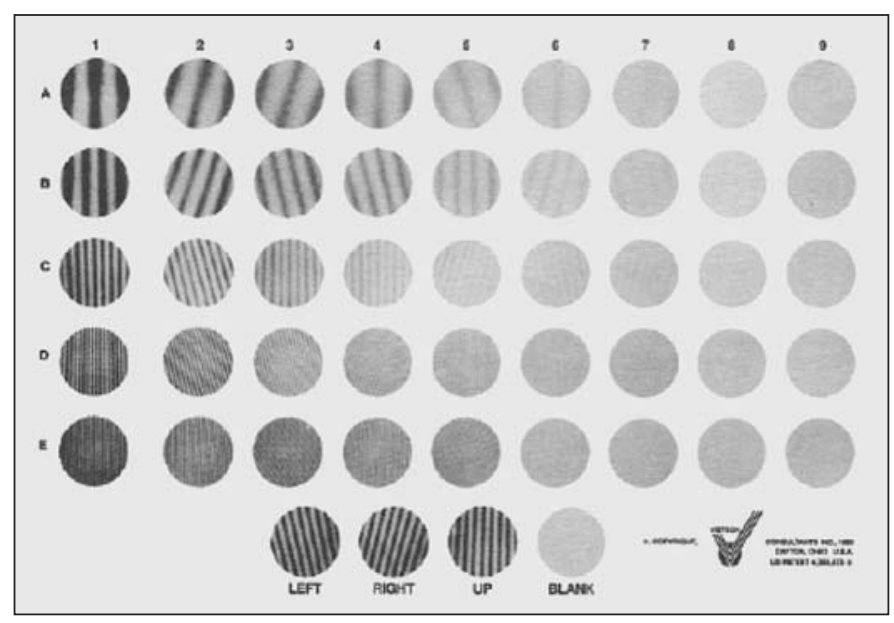

Figura 1 - Teste de sensibilidade ao contraste (VCTS 6500, Vistech Consultants Inc)

\begin{tabular}{|c|c|c|c|c|c|c|c|c|}
\hline $\begin{array}{l}\text { Freqüências } \\
\text { espaciais }\end{array}$ & 1 & 2 & 3 & 4 & 5 & 6 & 7 & 8 \\
\hline $1,5(\mathrm{~A})$ & 3 & 7 & 12 & 20 & 35 & 70 & 120 & 170 \\
\hline $3,0(B)$ & 4 & 9 & 15 & 24 & 44 & 85 & 170 & 220 \\
\hline $6,0(\mathrm{C})$ & 4 & 11 & 21 & 45 & 70 & 125 & 185 & 260 \\
\hline 12,0 (D) & 5 & 8 & 15 & 32 & 55 & 88 & 125 & 170 \\
\hline $18,0(E)$ & 4 & 7 & 10 & 15 & 26 & 40 & 65 & 90 \\
\hline
\end{tabular}


O estudo foi desenvolvido entre março a novembro de 2005, sendo aprovado pela Comissão de Ética para Análise de Projetos e Pesquisa da Diretoria Clínica do HC-FMUSP e todos os pacientes assinaram o termo de consentimento livre e esclarecido.

\section{RESULTADOS}

A amostra foi composta por 28 pacientes, sendo todos portadores de DM tipo 2. A idade variou entre 45 e 77 anos (média de $57,8 \pm 8,0$ ), sendo 19 pacientes $(67,9 \%)$ do sexo masculino e $9(32,1 \%)$ do feminino. Quanto ao tipo de retinopatia, $18(64,3 \%)$ apresentavam RD proliferativa e $10(35,7 \%)$, RD não proliferativa muito grave. Não foi observada nenhuma alteração na acuidade visual no pós-tratamento. Quanto à sensibilidade ao contraste, não houve alteração estatisticamente significante entre o pré e pós-tratamento em todas freqüências espaciais avaliadas: $1,5(\mathrm{p}=0,191) ; 3,0(\mathrm{p}=0,850) ; 6,0(\mathrm{p}=0,374)$; $12,0(\mathrm{p}=0,674)$ e $18,0(\mathrm{p}=0,443)$. As análises quantitativas para cada freqüência espacial estão demonstradas na tabela 2.

\section{DISCUSSÃO}

Para indivíduos normais, a sensibilidade ao contraste e acuidade visual são correlatas; entretanto, em alguns tipos de disfunções visuais encontradas (por exemplo no glaucoma, na catarata, na neurite óptica e na retinopatia diabética) podem cursar com diminuição da sensibilidade ao contraste, apesar da acuidade visual não se alterar ${ }^{(16)}$.

Por meio do Cambridge Low Contrast Sensitivity Chart, estudos evidenciaram redução temporária da sensibilidade ao contraste após 1-2 semanas da panfotocoagulação e a mesma retornou aos limiares anteriores ao tratamento, após 3 meses da aplicação do laser. Essa redução temporária foi justificada pelo efeito ofuscante do laser e pela disrupção e desorientação dos fotorreceptores ${ }^{(17)}$.

Em pacientes com RD tratada previamente com laser foi verificado que a sensibilidade ao contraste, com e sem ofus- camento, foi menor que no grupo não tratado. Além disso, o tempo de recuperação visual ao limiar prévio, no teste de "photostress", foi maior nos pacientes submetidos ao tratamento ${ }^{(18)}$. Outro estudo demonstrou que este retardo é devido à perda completa dos segmentos externos dos fotorreceptores, evidenciada após 6 meses da aplicação do laser ${ }^{(19)}$.

No presente estudo, a sensibilidade ao contraste foi avaliada em portadores de RD com acuidade visual de 20/20, submetidos à panfotocoagulação com laser de argônio. Como estudos prévios não estabeleceram acuidade de 20/20 como critério de inclusão, os resultados desses trabalhos podem ter sido afetados por esta variável, já que foi evidenciado que a fóvea possui maior percepção às freqüências espaciais elevadas e a retina periférica, às baixas. Não foram observadas alterações estatisticamente significantes entre o pré e pós-tratamento em todas freqüências espaciais avaliadas, denotando uma preservação da sensibilidade ao contraste após a panfotocoagulação. O tempo de seguimento adotado de três meses, aparentemente curto, foi estabelecido para obter-se uma correlação temporal com o tratamento.

Os novos padrões de exigência e qualidade farão com que o teste de sensibilidade ao contraste seja mais praticado não apenas em pesquisas, mas também na clínica oftalmológica rotineira, detectando alterações subclínicas mais precoces possíveis. $\mathrm{Na} \mathrm{RD}$, a panfotocoagulação com laser de argônio se faz necessária, muitas vezes, sem alterações quantitativas e/ou qualitativas da visão. Daí a importância que estudos demonstrem o grau de iatrogenia causado por este tratamento para, assim, serem avaliados os riscos e benefícios baseados em evidências.

\section{CONCLUSÃO}

Não foi evidenciada alteração significante na sensibilidade ao contraste de portadores de retinopatia diabética e acuidade de 20/20, após panfotocoagulação com laser de argônio. A importância disto se deve ao fato de que os estudos levam em consideração, na maioria das vezes, a visão quantitativa, podendo não estar alterada na vigência do tratamento. Assim,

Tabela 2. Sensibilidade ao contraste para cada freqüência espacial no pré e pós-panfotocoagulação de portadores de retinopatia diabética. Hospital das Clínicas da Universidade de São Paulo - 2005 ( $n=28$ pacientes)

\begin{tabular}{|c|c|c|c|c|c|c|c|}
\hline $\begin{array}{l}\text { Freqüências } \\
\text { espaciais }\end{array}$ & Momento & Média & $d p^{*}$ & Mínimo & Máximo & Mediana & $p^{\star \star}$ \\
\hline 1,5 & $\begin{array}{l}\text { Pré } \\
\text { Pós }\end{array}$ & $\begin{array}{l}20,71 \\
18,61\end{array}$ & $\begin{array}{l}6,68 \\
6,12\end{array}$ & $\begin{array}{r}12 \\
7\end{array}$ & $\begin{array}{l}35 \\
35\end{array}$ & $\begin{array}{l}20,00 \\
20,00\end{array}$ & 0,191 \\
\hline 3,0 & $\begin{array}{l}\text { Pré } \\
\text { Pós }\end{array}$ & $\begin{array}{l}33,29 \\
33,36\end{array}$ & $\begin{array}{l}10,16 \\
11,07\end{array}$ & $\begin{array}{l}24 \\
15\end{array}$ & $\begin{array}{l}44 \\
44\end{array}$ & $\begin{array}{l}24,00 \\
34,00\end{array}$ & 0,850 \\
\hline 6,0 & $\begin{array}{l}\text { Pré } \\
\text { Pós }\end{array}$ & $\begin{array}{l}45,46 \\
48,93\end{array}$ & $\begin{array}{l}22,87 \\
21,60\end{array}$ & $\begin{array}{l}21 \\
21\end{array}$ & $\begin{array}{l}125 \\
125\end{array}$ & $\begin{array}{l}45,00 \\
45,00\end{array}$ & 0,374 \\
\hline 12,0 & $\begin{array}{l}\text { Pré } \\
\text { Pós }\end{array}$ & $\begin{array}{l}44,86 \\
44,54\end{array}$ & $\begin{array}{l}26,69 \\
26,07\end{array}$ & $\begin{array}{r}8 \\
15\end{array}$ & $\begin{array}{l}88 \\
88\end{array}$ & $\begin{array}{l}55,00 \\
43,50\end{array}$ & 0,674 \\
\hline 18,0 & $\begin{array}{l}\text { Pré } \\
\text { Pós }\end{array}$ & $\begin{array}{l}21,75 \\
20,25\end{array}$ & $\begin{array}{r}11,54 \\
9,76\end{array}$ & $\begin{array}{l}4 \\
4\end{array}$ & $\begin{array}{l}40 \\
40\end{array}$ & $\begin{array}{l}26,00 \\
26,00\end{array}$ & 0,443 \\
\hline
\end{tabular}


sugerem-se estudos que também abordem as alterações qualitativas para melhor esclarecer iatrogenia causada pela panfotocoagulação com laser.

\section{ABSTRACT}

Purpose: To evaluate contrast sensitivity in patients with diabetic retinopathy (DR) treated with argon laser panphotocoagulation. Methods: Prospective study of patients with diabetic retinopathy and 20/20 visual acuity, treated with retinal panphotocoagulation, following ETDRS criteria. The patients were submitted, initially, to complete ophthalmologic evaluation and contrast sensitivity testing (Vision Contrast Test System). After 3 months of treatment, they were reevaluated by means of visual acuity and contrast sensitivity. Results: The sample comprised 28 patients ( 28 eyes), all with type II diabetes. A ranged from 45 to 77 years (mean $57.8 \pm 8.0), 19(67.9 \%)$ patients were male and $9(32.1 \%)$ female. Regarding the type of retinopathy, 18 (64.3\%) had proliferative DR and $10(35.7 \%)$ very severe non proliferative RD. No visual acuity alteration was observed after treatment. In relation to contrast sensitivity, there were no alterations between pre and post-treatment in all evaluated spacial frequencies $1.5(p=0.191) ; 3.0(p=0.850) ; 6.0$ $(p=0.374) ; 12.0(p=0.674)$ e $18.0(p=0.443)$. Conclusion: There was no significant alteration in the contrast sensitivity of patients with diabetic retinopathy after panphotocoagulation with argon laser in the studied period.

Keywords: Diabetic retinopathy; Diabetic retinopathy/therapy; Laser coagulation; Diabetes mellitus/complications; Visual acuity

\section{REFERÊNCIAS}

1. Early photocoagulation for diabetic retinopathy. ETDRS report number 9 . Early Treatment Diabetic Retinopathy Study Research Group. Ophthalmology. 1991;98(5 Suppl):766-85.
2. Early Treatment Diabetic Retinopathy Study design and baseline patient characteristics. ETDRS report number 7. Ophthalmology. 1991;98(5 Suppl):741-56.

3. McDonald HR, Schatz H. Macular edema following panretinal photocoagulation. Retina. 1985;5(1):5-10.

4. Henricsson M, Heijl A. The effect of panretinal photocoagulation on visual acuity, visual fields and on subjective visual impairment in preproliferative and early proliferative diabetic retinopathy. Acta Ophthalmol (Copenh). 1994; 72(5):570-5.

5. Mäntyjärvi M. Colour vision and dark adaptation in diabetic patients after photocoagulation. Acta Ophthalmol (Copenh). 1989;67(2):113-8.

6. Pender PM, Benson WE, Compton H, Cox GB. The effects of panretinal photocoagulation on dark adaptation in diabetics with proliferative retinopathy. Ophthalmology. 1981;88(7):635-8.

7. Shah KB, Han DP. Proliferative diabetic retinopathy. Int Ophthalmol Clin. 2004;44(4):69-84.

8. Perlman I, Gdal-On M, Miller B, Zonis S. Retinal function of the diabetic retina after argon laser photocoagulation assessed electroretinographically. $\mathrm{Br}$ J Ophthalmol. 1985;69(4):240-6.

9. Wolfe JM. An introduction to contrast sensitivity testing. In: Nadler MP, Miller D, Nadler DJ, editors. Glare and contrast sensitivity for clinicians. New York: Springer-Verlag; 1990. p.5-23.

10. Monteiro MLR. Contribuição ao estudo da sensibilidade ao contraste em portadores de defeitos campimétricos atribuídos a tumores hipofisários [tese]. São Paulo: Faculdade de Medicina da Universidade de São Paulo; 1992.

11. Campbell FW, Maffei L. Contrast and spatial frequency. Sci Am. 1974;231 (5):106-14.

12. Arden GB. The importance of measuring contrast sensitivity in cases of visual disturbance. Br J Ophthalmol. 1978;62(4):198-209.

13. McDonald HR, Schatz H. Visual loss following panretinal photocoagulation for proliferative diabetic retinopathy. Ophthalmology. 1985;92(3):388-93.

14. Shimura M, Yasuda K, Nakazawa T, Tamai M. Visual dysfunction after panretinal photocoagulation in patients with severe diabetic retinopathy and good vision. Am J Ophthalmol. 2005;140(1):8-15.

15. Techniques for scatter and local photocoagulation treatment of diabetic retinopathy: Early Treatment Diabetic Retinopathy Study Report nº. 3. The Early Treatment Diabetic Retinopathy Study Research Group. Int Ophthalmol Clin. 1987;27(4):254-64.

16. Marmor MF. Contrast sensitivity versus visual acuity retinal disease. Br J Ophthalmol. 1986;70(7):553-9.

17. Khosla PK, Rao V, Tewari HK, Kumar A. Contrast sensitivity in diabetic retinopathy after panretinal photocoagulation. Ophthalmic Surg. 1994;25(8): 516-20.

18. Lövestam-Adrian M, Svendenius N, Agardh E. Contrast sensitivity and visual recovery time in diabetic patients treated with panretinal photocoagulation. Acta Ophthalmol Scand. 2000;78(6):672-6.

19. Stitt AW, Gardiner TA, Archer DB. Retinal and choroidal responses to panretinal photocoagulation: an ultrastructural perspective. Graefes Arch Clin Exp Ophthalmol. 1995;233(11):699-705. Comment in: Graefes Arch Clin Exp Ophthalmol. 1996;234(5):349. 